\title{
Prevalência de tuberculose na população privada de liberdade: revisão sistemática e metanálise
}

\author{
Tiago Ricardo Moreira, ${ }^{1}$ Aline Campos Lemos, ${ }^{2}$ Renata Maria Colodette, ${ }^{1}$ \\ Andréia Patrícia Gomes ${ }^{3}$ e Rodrigo Siqueira Batista ${ }^{4}$
}

Como citar Moreira TR, Lemos AC, Colodette RM, Gomes AP, Batista RS. Prevalência de tuberculose na população privada de liberdade: revisão sistemática e metanálise. Rev Panam Salud Publica. 2019;43:e16. https:/ doi.org/10.26633/RPSP.2019.16

RESUMO Objetivo. Estimar a prevalência de tuberculose entre a população privada de liberdade. Métodos. Realizou-se uma revisão sistemática com metanálise. Foram selecionados estudos publicados de janeiro de 1997 a dezembro de 2016 nas bases de dados MEDLINE (via PubMed), SciELO e LILACS. O termos de busca foram ("tuberculosis"[MeSH] OR "tuberculosis"[TIAB]) AND ("prisons"[MeSH] OR "prisons"[TIAB]); ou ("tuberculose" [DeCS] OU "tuberculose" [palavras] E "prisões" [DeCS] OU "prisões" [palavras]). O desfecho primário foi a prevalência de tuberculose com intervalo de confiança de 95\% (IC95\%). Na metanálise, as variáveis associadas com as taxas de prevalência de tuberculose na população privada de liberdade na análise univariada $(\mathrm{P} \leq 0,20)$ foram incluídas no modelo final multivariado.

Resultados. Com base nos 29 estudos incluídos na metanálise, 2163 presos foram identificados com tuberculose. A prevalência combinada de tuberculose entre os prisioneiros foi de $2 \%$ (IC95\%: 0,02 a 0,02). A prevalência de tuberculose entre prisioneiros em países com prevalência de 0 a 24 por 100 mil habitantes na população geral ficou abaixo de $1 \%$ (IC95\% = 0,00 a 0,00). Nos países com prevalência de tuberculose de 25 a 99/100 mil, a estimativa foi de 3\% (IC95\% = 0,02 a 0,04); e nos países com prevalência $\geq 300 / 100$ mil, a estimativa foi de $8 \%$ $($ IC95\% $=0,05$ a 0,11$)$.

Conclusão. O presente estudo reafirma a alta magnitude da tuberculose entre a população privada de liberdade no contexto mundial. Os resultados mostram ainda uma ligação entre a prevalência de tuberculose na população geral e a prevalência da doença dentro dos presídios.

Palavras-chave Tuberculose; prisões; prevalência; metanálise.

\footnotetext{
Universidade Federal de Viçosa (UFV), Programa de Pós-Graduação em Ciências da Saúde, Departamento de Medicina e Enfermagem, Viçosa (MG), Brasil. Correspondência: Tiago Ricardo Moreira, tiago.ricardo@ufv.br

2 Hospital São João Batista, Viçosa (MG), Brasil.

Fundação Oswaldo Cruz (FIOCRUZ), Escola Nacional de Saúde Pública Sérgio Arouca, Programa de Pós-graduação em Bioética, Ética Aplicada e Saúde Coletiva, Rio de Janeiro (RJ), Brasil.

4 Universidade Federal do Rio de Janeiro (UFRJ), Programa de Pós-graduação em Bioética, Ética Aplicada e Saúde Coletiva, Rio de Janeiro (RJ), Brasil.
}

A ocorrência de tuberculose ativa nas prisões é geralmente relatada como sendo muito maior do que aquela da população geral correspondente (1). Mesmo assim, as prisões são muitas vezes ignoradas pelos setores nacionais de saúde e não estão incluídas nas estatísticas nacionais (2). Nos ambientes frequentemente lotados das prisões, onde pessoas com e sem tuberculose estão em contato, aumentam os riscos de contrair e desenvolver tuberculose resistente a vários medicamentos (3). Além disso, as prisões representam um reservatório de transmissão de doenças para a comunidade em geral. Sendo assim, o controle efetivo da tuberculose nesse ambiente pode impedir que a infecção se espalhe

Este é um artigo de acesso aberto distribuído sob os termos da Licença Creative Commons Attribution-NonCommercial-NoDerivs 3.0 IGO, que permite o uso, distribuiç̃o e reproducão em qualquer meio, desde que o trabalho original seja devidamente citado. Não são permitidas modificaçoes ou uso comercial dos artigos. Em qualquer reprodução do artigo, não deve haver nenhuma sugestão de que a OPAS ou o artigo avaliem qualquer organização ou produtos específicos. Não é permitido o uso do logotipo da OPAS. Este aviso deve ser preservado juntamente com o URL original do artigo. 
para a população geral através de funcionários, visitantes e contatos próximos dos prisioneiros libertados (4).

Diferentes fatores podem ser atribuídos à maior prevalência de tuberculose nas prisões. Um estudo com prisioneiros etíopes mostrou que era pobre o conhecimento dos prisioneiros sobre as causas da doença (5). Outros estudos com prisioneiros relataram associação entre risco de desenvolver tuberculose e desnutrição (6-8), analfabetismo $(5,9,10)$, tabagismo $(5,11,12)$, tempo de encarceramento $(5,7,13)$, superlotação e ventilação inadequada $(6,14,15)$, idade produtiva (12), residência urbana antes da prisão (11), história de contato com pacientes com tuberculose (16) e infecção anterior pela tuberculose (17).

A tuberculose não é uma consequência inevitável do encarceramento e pode ser controlada através de programas fundamentados na estratégia global para enfrentamento da tuberculose proposta pela Organização Mundial da Saúde (OMS) (18). O rastreio sistemático de contatos e grupos de alto risco é um dos pilares dessa estratégia, mas muitos sistemas penitenciários enfrentam uma variedade de desafios que impedem o controle da tuberculose. Tais desafios incluem, entre outros, capacidade laboratorial e ferramentas de diagnóstico insuficientes, interrupções no fornecimento de medicamentos, fraca integração entre serviços de tuberculose dos sistemas de saúde e da prisão, medidas inadequadas de controle de infecção e baixa prioridade política para atenção à saúde na prisão (2).

Sendo assim, o objetivo do presente estudo foi estimar a prevalência de tuberculose entre a população privada de liberdade com base nos dados disponíveis na literatura.

\section{MATERIAIS E MÉTODOS}

Realizou-se uma revisão sistemática com metanálise. O protocolo da revisão foi registrado na base International Prospective Register of Systematic Reviews (PROSPERO) sob o número CRD42018084284. O estudo foi realizado de acordo com as recomendações PRISMA (19).

\section{Critérios de elegibilidade}

Foram elegíveis para inclusão estudos originais que relatassem a incidência ou prevalência de tuberculose pulmonar nas prisões ou que relatassem o número de casos de tuberculose identificados no estudo, juntamente com o número total de presos ou pessoas-anos de acompanhamento. Os estudos com delineamento de coorte (casos incidentes) não foram excluídos de nossa análise por apresentarem dados da população de referência e dados da prevalência de tuberculose na linha de base, tornando possível o cálculo da prevalência no período do estudo. Para casos de incidentes e prevalentes de tuberculose, foram incluídos diagnósticos definitivos (confirmados microbiologicamente por cultura ou baciloscopia) e presuntivos (com base em critérios clínicos, de imagem) ou por biologia molecular. Para incluir estudos de qualidade comparável, consideraram-se apenas dados publicados em revistas com revisão por pares.

Foram excluídos estudos com as seguintes características: 1) relatórios que apresentavam apenas série temporal; 2) relatos limitados a investigações de surtos; 3) estudos relatando apenas prevalência de infecção tuberculosa latente (ILTB); 4) estudos voltados apenas para tuberculose resistente a vários medicamentos; 5) estudos de caso-controle; 6) estudos que iniciaram a coleta de dados antes de 1990 (critério utilizado apenas para estudos longitudinais); 7) estudos realizados apenas com funcionários das prisões; 8) estudos que incluíram menores de 18 anos; e 9) estudos realizados com dados secundários. Todas as citações duplicadas foram eliminadas do banco de dados inicial.

Não houve restrição quanto ao idioma de publicação para os estudos identificados. Foram selecionados estudos publicados de janeiro de 1997 a dezembro de 2016.

\section{Fontes de informações e estratégias de busca}

A busca pelos artigos foi realizada nas seguintes bases de dados: MEDLINE (via PubMed) (https://www.ncbi.nlm.nih. gov/pubmed/), Scientific Electronic Library Online (SciELO) (http://www. scielo.br/) e Literatura Latino-Americana e do Caribe em Ciências da Saúde (LILACS) (http://lilacs.bvsalud.org/). A estratégia de busca para o MEDLINE foi a seguinte: ("tuberculosis"[Mesh] OR "tuberculosis"[TIAB]) AND ("prisons" [Mesh] OR "prisons"[TIAB]). Para as bases LILACS e SciELO utilizou-se a combinação de palavras/termos: ("tuberculose" [DeCS] OU "tuberculose" [palavras] E "prisões" [DeCS] OU "prisões" [palavras]).

As pesquisas foram realizadas a partir de fevereiro de 2017. A última busca foi em março de 2017. Adicionalmente, foram examinadas as listas de referências bibliográficas dos estudos relevantes para identificar outros artigos potencialmente elegíveis.

\section{Seleção dos estudos e extração dos dados}

Os artigos incluídos no estudo foram selecionados por dois autores (LA e MT) em quatro passos. No primeiro passo, os artigos foram identificados por meio da busca eletrônica, organizados e revisados para verificação de duplicatas pelos dois autores de forma independente. A concordância entre os revisores foi alta (kappa $=0,85)$ e as divergências em todos os passos da seleção dos estudos foram resolvidas por consenso.

No segundo passo, os dois autores analisaram de forma independente os títulos dos artigos. Foram excluídos os artigos que preenchiam algum critério de exclusão. Havendo insuficiência de dados, o resumo era deixado para a próxima etapa de avaliação.

No terceiro passo, os resumos dos artigos selecionados no segundo passo foram examinados. Os artigos que não continham características da população estudada foram excluídos. Havendo insuficiência de dados, o resumo era deixado para a próxima etapa de avaliação.

Finalmente, no quarto passo, os textos completos dos artigos selecionados até o momento foram recuperados e revisados. Foram selecionados os que apresentavam dados de prevalência na população carcerária dos estudos ou que apresentavam dados que permitissem esse cálculo.

Foi definido um protocolo para a extração de dados dos textos completos. Essa extração foi realizada por dois autores (LA e TR). As discordâncias foram decididas por consenso entre os dois. Os seguintes dados foram extraídos e categorizados: título, local de estudo (América do Sul, Europa, Ásia, África), ano de publicação (2000 a 2010, 2011 a 2016), ano de coleta de dados de estudo, delineamento do estudo (incidência ou prevalência), fontes de dados, número de centros prisionais (um centro ou mais de 
um centro), tamanho da amostra $(\leq 1050$; 1051 a 2 300; 2301 a $6111 ; 6119$ ou mais participantes), número de casos de tuberculose e/ou prevalência/incidência, IC e método de diagnóstico de tuberculose (bacterioscopia, radiológico e bacterioscopia, biologia molecular). A prevalência de tuberculose na população geral do país de cada estudo foi obtida do relatório global de tuberculose de 2017 (0 a 24 casos por $100 \mathrm{mil}, 25$ a 99 casos por 100 mil, 100 a 199 casos por 100 mil, 200 a 299 casos por 100 mil e 300 ou mais casos por $100 \mathrm{mil}$ ) (18).

Após essa extração, uma nova revisão dos artigos selecionados foi realizada para assegurar que os dados de cada indivíduo não fossem inseridos na análise mais de uma vez. Artigos que não apresentassem todas as informações tiveram o autor contatado pelo menos duas vezes para os devidos questionamentos. Para gerenciamento das referências foi utilizado o programa EndNote X7.

\section{Avaliação da qualidade metodológica dos estudos incluídos}

O instrumento de avaliação crítica de estudos de prevalência proposto por Loney et al. (20) foi utilizado, com adaptações, para determinar a qualidade dos artigos. Os autores adotam oito critérios: 1) amostragem probabilística ou censitária; 2) fonte de amostragem adequada (censo oficial, censo escolar, entre outros); 3) tamanho da amostra previamente calculado; 4) forma de aferição adequada (utilizando equipamento validado); 5) aferição imparcial realizada por avaliadores treinados; 6) taxa de resposta adequada $(>70,0 \%)$ e descrição das recusas; 7$)$ apresentação dos IC e das análises de subgrupos de interesse; e 8) sujeitos do estudo bem descritos e adequados à pergunta de pesquisa.

Para cada critério atendido o estudo recebeu um ponto. Foram considerados de alta qualidade os estudos com pontuação 7 e 8; moderada qualidade, 4 a 6 pontos; e de baixa qualidade, 0 a 3 pontos (19). A avaliação da qualidade não foi utilizada como critério de exclusão dos artigos, sendo entretanto parâmetro para o estudo da heterogeneidade e da análise de subgrupos.

A opção pela escala de Loney et al. (20) se deu porque a mesma avalia pesquisas sobre prevalência ou incidência (demandando adaptações ao tipo de desfecho que está sendo esperado), é de fácil aplicação e permite análises quantitativas. Além disso, a pontuação obtida pode ser usada para comparar os artigos incluídos na revisão quanto à qualidade.

\section{Análise dos dados}

O desfecho primário foi a prevalência de tuberculose com IC95\%. Foram estimadas as medidas sumárias para a população total e subgrupos definidos por delineamento do estudo, local do estudo, ano de publicação, número de centros prisionais, tamanho da amostra, prevalência de tuberculose na população geral de cada país, método diagnóstico e qualidade dos estudos.

A metanálise foi realizada usando um modelo de efeito randômico. A heterogeneidade foi avaliada pelo teste do qui-quadrado $\left(\chi^{2}\right)$ com significância de $P<0,10$, sendo sua magnitude apurada pelo o I-quadrado $\left(\mathrm{I}^{2}\right)(21)$. As análises foram realizadas com os comandos Metaprop e Metareg do programa Stata (versão 11.0). Foram realizadas metarregressões com o objetivo de identificar as causas da heterogeneidade, utilizando o teste de Knapp e Hartung (22). Inicialmente, foi feita uma análise univariada. Todas as variáveis associadas com as taxas de prevalência de tuberculose na população privada de liberdade nessa análise $(P \leq 0,20)$ foram incluídas no modelo final multivariado. Para essas análises, um nível de significância de 5\% foi estabelecido. Avaliou-se ainda a existência do efeito de estudos pequenos (small-study effect) por meio de inspeção visual do gráfico de funil e do teste de Egger (23).

\section{RESULTADOS}

A figura 1 descreve o processo de seleção de artigos. Foram triados 377 trabalhos, sendo que 280 foram excluídos a

\section{FIGURA 1. Fluxograma de seleção de artigos sobre tuberculose na população privada de liberdade}

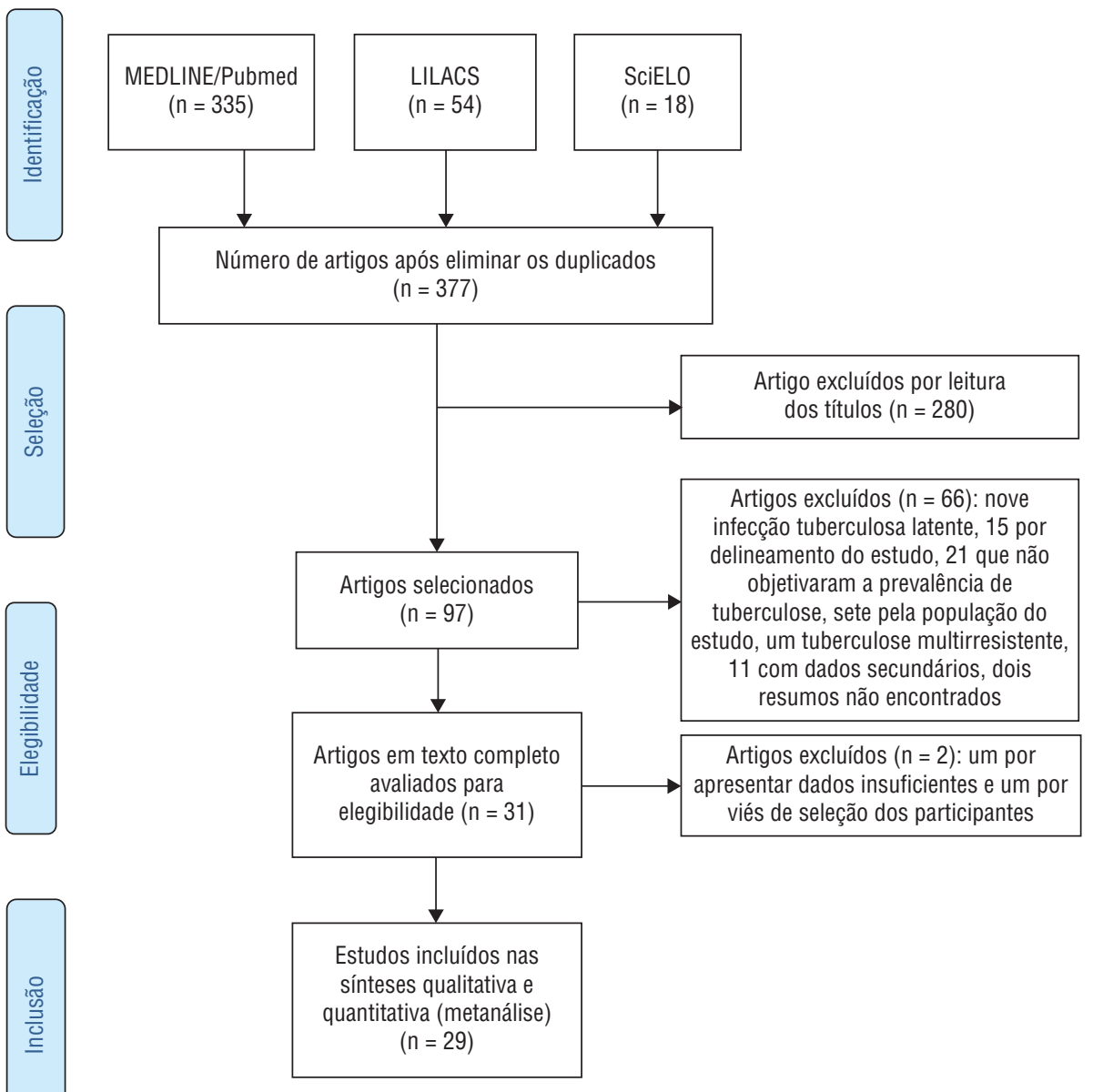


partir da leitura dos títulos. Após as avaliações iniciais, foram revisados 97 resumos e, a partir deles, 31 artigos completos foram analisados, dos quais 29 foram incluídos nesta revisão sistemática (5-16, 24-40). Nenhum estudo foi incluído na análise após exame das listas de referências dos artigos revisados. Os estudos investigaram 151181 indivíduos privados de liberdade e 2163 casos de tuberculose foram identificados. Informações para a identificação dos estudos selecionados para esta metanálise encontram-se na tabela 1. A tabela 2 mostra as principais características dos estudos. A população dos estudos variou de 168 prisioneiros na Nigéria (27) a 69859 na Tailândia (32).

Os 29 estudos incluídos foram avaliados quanto à heterogeneidade e ao viés de publicação. A análise mostrou uma heterogeneidade substancial do teste $\mathrm{Q}$ $(P<0,001)$ e estatística $\mathrm{I}^{2}\left(\mathrm{I}^{2}=98,42 \%\right)$. A figura 2 mostra assimetria entre as investigações, o que foi confirmado pelo teste de Egger $(P<0,001)$, apontando a probabilidade de estudos pequenos, que possivelmente encontraram prevalências baixas, não terem sido publicados.

A prevalência agrupada de tuberculose entre indivíduos da população privada de liberdade foi de $\%$ (IC95\%: 0,02 a 0,02$)$ no modelo randômico. A heterogeneidade entre os estudos foi significativa $(P<0,001)$ (figura 3$). \mathrm{Na}$ análise univariada, as variáveis significativamente associadas com diferenças nas taxas prevalência de tuberculose na população privada de liberdade $(P \leq 0,2)$ foram: delineamento do estudo, número de centros prisionais e prevalência de tuberculose da população geral. Assim, essas variáveis foram incluídas no modelo final da metarregressão. No modelo final, as prevalências de tuberculose na população geral entre 25 e $99 / 100 \mathrm{mil}$ $(P=0,042)$ e acima de 300/100 mil $(P<0,001)$ mantiveram-se associadas com prevalência da tuberculose na população privada de liberdade (tabela 2).

TABELA 1. Identificação dos artigos selecionados para metanálise sobre tuberculose na população privada de liberdade

\begin{tabular}{|c|c|c|c|c|c|c|}
\hline Autor & Ano & Local & Delineamento & Amostra & Casos & Prevalência (\%) \\
\hline$\overline{\text { Abele et al. (5) }}$ & 2011 & Etiópia & Prevalência & 2300 & 44 & 1,91 \\
\hline Aerts et al. (13) & 2000 & Geórgia & Prevalência & 7473 & 448 & 6,00 \\
\hline Aguilera et al. (7) & 2016 & Chile & Incidência & 26644 & 33 & 0,12 \\
\hline Ali et al. (16) & 2015 & Etiópia & Prevalência & 15495 & 71 & 0,46 \\
\hline Banda et al. (24) & 2009 & Malawi & Prevalência & 7661 & 54 & 0,70 \\
\hline Banu et al. (25) & 2010 & Bangladesh & Prevalência & 11001 & 245 & 2,23 \\
\hline Carbone et al. (26) & 2015 & Brasil & Prevalência & 3380 & 31 & 0,92 \\
\hline Chigbu et al. (27) & 2010 & Nigéria & Incidência & 168 & 3 & 1,79 \\
\hline Estevan et al. (28) & 2013 & Brasil & Prevalência & 249 & 1 & 0,40 \\
\hline Fuge et al. (29) & 2016 & Etiópia & Prevalência & 859 & 3 & 0,35 \\
\hline Habeenzu et al. (8) & 2007 & Zâmbia & Prevalência & 6118 & 245 & 4,01 \\
\hline Hanau-Berçot et al. (30) & 2000 & França & Incidência & 31546 & 68 & 0,22 \\
\hline Henostroza et al. (31) & 2013 & Zâmbia & Prevalência & 2323 & 228 & 9,81 \\
\hline Jittimanee et al. (32) & 2007 & Tailândia & Prevalência & 71594 & 254 & 0,35 \\
\hline Kazi et al. (33) & 2010 & Paquistão & Prevalência & 365 & 7 & 2,20 \\
\hline Kuhleis et al. (10) & 2012 & Brasil & Prevalência & 1900 & 72 & 4,96 \\
\hline Leung et al. (34) & 2005 & Hong Kong & Prevalência & 814 & 10 & 1,23 \\
\hline Martin et al. (35) & 2001 & Espanha & Incidência & 1050 & 23 & 0,64 \\
\hline Moges et al. (36) & 2012 & Etiópia & Prevalência & 1754 & 26 & 1,48 \\
\hline Nogueira et al. (37) & 2012 & Brasil & Prevalência & 2408 & 20 & 0,83 \\
\hline Öngen et al. (6) & 2013 & Turquia & Prevalência & 4615 & 5 & 0,11 \\
\hline Paião et al. (15) & 2016 & Brasil & Incidência & 1422 & 25 & 2,46 \\
\hline Pendzich et al. (14) & 2015 & Polônia & Prevalência & 720 & 11 & 1,53 \\
\hline Rao (38) & 2004 & Paquistão & Prevalência & 4870 & 32 & 0,66 \\
\hline Sanchez et al. (9) & 2005 & Brasil & Prevalência & 1052 & 48 & 4,56 \\
\hline Sretrirutchai et al. (39) & 2002 & Tailândia & Prevalência & 4751 & 27 & 0,57 \\
\hline Telisinghe et al. (11) & 2014 & África do Sul & Prevalência & 968 & 34 & 3,51 \\
\hline Valença et al. (12) & 2015 & Brasil & Prevalência & 764 & 36 & 4,71 \\
\hline Winetsky et al. (40) & 2014 & Tajiquistão & Prevalência & 1317 & 59 & 4,48 \\
\hline
\end{tabular}

Na figura 3 encontram-se também as prevalências agrupadas de tuberculose em indivíduos da população privada de liberdade de acordo com os estratos de prevalência na população geral do país. A figura mostra que, para países com prevalência de 0 a 24 por 100 mil, a estimativa de prevalência foi abaixo de $1 \%$ (IC95\%: 0,00 a 0,00). Para prevalência de 25 a 99 por 100 mil, a estimativa foi de $3 \%$ (IC95\%: 0,02 a 0,04), e para prevalência $\geq 300$ por 100 mil, a estimativa foi de $8 \%$ (IC95\%: 0,05 a 0,11).

\section{DISCUSSÃO}

A maioria dos estudos incluídos nesta revisão relatou taxas de prevalência de tuberculose em prisioneiros muito superiores às encontradas na população geral dos países onde se localizavam os presídios. Foram identificados estudos de quatro continentes, com ampla variação na prevalência de tuberculose - desde 0 a 24 casos por 100 mil habitantes até 300 ou mais casos de tuberculose por $100 \mathrm{mil}$ habitantes (18). Os estudos utilizaram vários critérios de inclusão, métodos de diagnóstico, investigaram casos suspeitos a partir do rastreio de sintomáti$\cos$ e não incluíram os pacientes tratados anteriormente.

A tuberculose nas prisões é muitas vezes ignorada e continua a ser um importante problema de saúde pública em muitos contextos, particularmente em países com alta incidência dessa doença (2). A estimativa de prevalência combinada de tuberculose entre prisioneiros foi de $2 \%$ (IC95\%: 0,02 a 0,02; $P<0,001$ ), sendo 14 vezes maior que a prevalência encontrada na população geral $(0,14 \%)$ (18). Resultados análogos foram relatados por Bussano et al. (1) em uma revisão sistemática sobre incidência de tuberculose nas prisões. Outra revisão sistemática que enfocou prisões da Etiópia mostrou uma prevalência combinada de tuberculose entre os prisioneiros de $8,33 \%$ (41). Uma terceira revisão também indicou alta prevalência de tuberculose nas prisões, relatada como sendo 3 a 1000 vezes maior do que na população civil. Além disso, altos níveis de tuberculose multirresistente e extensivamente resistente a drogas (XDR) foram relatados nas prisões, o que é um alerta para a revisão das estratégias de controle (42). Esses números elevados podem estar associados às más condições da maioria 
TABELA 2. Distribuição das principais características dos estudos sobre tuberculose na população privada de liberdade e associação com as estimativas de prevalência de tuberculose nessa população

\begin{tabular}{|c|c|c|c|c|c|}
\hline \multirow{3}{*}{ Variáveis } & \multirow{3}{*}{ No. } & \multirow{3}{*}{$\%$} & \multirow{3}{*}{$\begin{array}{c}\text { Prevalência } \\
\%\end{array}$} & \multicolumn{2}{|c|}{ Metarregressão $0^{\mathrm{a}}$} \\
\hline & & & & Univariada & Multivariada \\
\hline & & & & $P$-valor & $P$-valor \\
\hline \multicolumn{6}{|l|}{ Ano de publicação do estudo } \\
\hline 2000 a 2010 & 13 & 44,8 & 2,30 & Ref & - \\
\hline 2011 a 2016 & 16 & 55,2 & 2,62 & 0,940 & - \\
\hline \multicolumn{6}{|l|}{ Delineamento do estudo } \\
\hline Prevalência & 24 & 82,8 & 2,71 & Ref & Ref \\
\hline Incidência & 5 & 17,2 & 1,37 & 0,200 & 0,845 \\
\hline \multicolumn{6}{|c|}{ Número de unidades prisionais estudadas } \\
\hline Uma & 14 & 48,3 & 3,22 & Ref & Ref \\
\hline Duas ou mais & 15 & 51,7 & 1,78 & 0,108 & 0,137 \\
\hline \multicolumn{6}{|l|}{ Tamanho da amostra } \\
\hline Até 1050 & 8 & 27,6 & 2,68 & Ref & - \\
\hline 1051 a 2300 & 7 & 24,1 & 3,04 & 0,900 & - \\
\hline 2301 a 6118 & 7 & 24,1 & 2,69 & 0,734 & - \\
\hline 6119 ou mais & 7 & 24,1 & 1,45 & 0,302 & - \\
\hline \multicolumn{6}{|l|}{ Métodos diagnósticos } \\
\hline Radiologia + bacterioscopia & 10 & 34,5 & 3,39 & 0,392 & - \\
\hline Bacterioscopia & 17 & 58,6 & 2,12 & Ref & - \\
\hline Biologia molecular & 2 & 6,9 & 0,91 & 0,458 & - \\
\hline \multicolumn{6}{|l|}{ Local do estudo } \\
\hline África & 9 & 31,0 & 3,44 & 0,436 & - \\
\hline América do Sul & 8 & 27,6 & 2,22 & 0,873 & - \\
\hline Ásia & 7 & 24,1 & 1,85 & 0,716 & - \\
\hline Europa & 5 & 17,2 & 2,02 & Ref & - \\
\hline \multicolumn{6}{|c|}{ Prevalência de tuberculose na população geral } \\
\hline 0 a $24 / 100 \mathrm{mil}$ & 5 & 17,2 & 0,85 & Ref & Ref \\
\hline 25 a 99/100 mil & 9 & 31,0 & 2,91 & $<0,001$ & 0,042 \\
\hline 100 a $199 / 100$ mil & 7 & 24,1 & 1,39 & 0,646 & 0,989 \\
\hline 200 a $299 / 100 \mathrm{mil}$ & 5 & 17,2 & 1,48 & 0,137 & 0,937 \\
\hline 300 ou mais/100 mil & 3 & 10,3 & 8,09 & $<0,001$ & 0,001 \\
\hline \multicolumn{6}{|l|}{ Qualidade dos estudos ${ }^{\mathrm{b}}$} \\
\hline Alta & 10 & 34,5 & 3,03 & Ref & - \\
\hline Moderada & 19 & 65,5 & 2,19 & 0,757 & - \\
\hline
\end{tabular}

${ }^{a}$ Ref: categoria de referência.

'Nenhum artigo foi classificado na categoria "baixa".

FIGURA 2. Gráfico de funil: prevalência de tuberculose na população privada de liberdade em cada estudo pelo desvio padrão dos estudos ${ }^{a}$

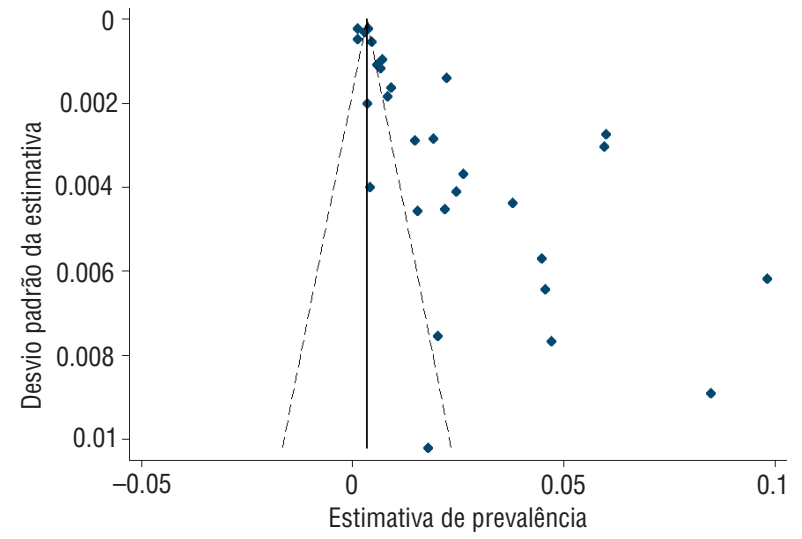

dos presídios: confinamento de grande número de casos, frequentemente bacilíferos, em celas na maioria das vezes coletivas, mal ventiladas, sem iluminação solar e superpopulosas, associado à insuficiência notória dos serviços de saúde penitenciária (43).

Uma alta heterogeneidade $\left(\mathrm{I}^{2}=98,42 \%\right)$ foi identificada entre os estudos e se manteve em todas as análises de subgrupos, o que demanda atenção com a extrapolação dos resultados. $\mathrm{Na}$ análise de metarregressão, a prevalência de tuberculose na população privada de liberdade mostrou-se associada à prevalência de tuberculose na população geral dos países onde se localizavam os presídios. A prevalência combinada de tuberculose em presídios de países com baixa prevalência na população geral $(0$ a 24/100 mil) ficou abaixo de 1\% (IC95\%: $0,00$ a 0,00$)$, enquanto a prevalência combinada de tuberculose em países com prevalência de tuberculose acima de 300/100 mil na população geral foi de $8 \%$ (IC $95 \%$ : 0,05 a 0,11). Além de causas metodológicas, a grande heterogeneidade entre os resultados dos estudos pode ter ocorrido devido às desigualdades sociais e negligência com a doença entre esses países (44).

As prevalências mais baixas de tuberculose nos estudos realizados em países com baixas estimativas na população geral poderiam ser atribuídas a fortes estratégias de controle da doença, baixas taxas de incidência e bons sistemas de saúde tanto nas prisões quanto na população em geral. Porém, mesmos em países com baixa prevalência de tuberculose, há de se considerar a exponencial diferença em relação às estimativas encontradas nos presídios, indicando que estratégias de controle precisam ser ainda mais particularizadas para que sejam efetivadas em todos os grupos populacionais (45).

Vale ressaltar que a tuberculose é uma enfermidade de cunho eminentemente social, e que sua persistência decorre de inadequadas condições sociais e iniquidades em saúde. Além de medidas de controle e detecção precoce, a superação da tuberculose decorrerá da superação das desigualdades sociais (46). A educação para identificação precoce da tuberculose, o gerenciamento precoce de casos e o tratamento adequado, o rastreio de presos na chegada, o isolamento de casos com esfregaços positivos dentro do quadro de serviços de saúde 
FIGURA 3. Prevalência agrupada de tuberculose na população privada de liberdade segundo a prevalência de tuberculose na população geral dos países ${ }^{a}$

\section{Estudo}

\section{0-199}

Abele et al., 2011

All et al., 2015

Fuge et al., 2016

JIttlmanee et al., 2007

Moges et al., 2012

Sanchez et al., 2005

Sretrirutchai et al., 2002

Subtotal $\left(\left.\right|^{\wedge} 2-93.34 \%, p-0,00\right)$

25-99

Aerts et al., 2000

Carbone et al., 2015

Estevan et al., 2013

Kuhleis et al., 2012

Leung et al., 2005

Noguelra et al., 2012

Palào et al., 2016

Valença et al., 2015

Winetsky et al., 2014

Subtotal (|^2 - 97.80\%, p - 0,00)

0-24

Agullera et al., 2016

Hanau-Berçot et al., 2000

Martin et al., 2001

Ongen et al., 2013

Pendzlch et al., 2015

Subtotal ( $\left.\left.\right|^{\wedge} 2-92.25 \%, p-0,00\right)$

\section{0-299}

Banda et al., 2009

Banu et al., 2010

Chigbu et al., 2010

Kazi et al., 2010

Rao, 2004

Subtotal $\left(\left.\right|^{\wedge} 2-95.86 \%, p-0,00\right)$

300 ou +

Habeenzu et al., 2007

Henostroza et al., 2013

Telisinghe et al., 2014

Subtotal $\left(\left.\right|^{\wedge} 2-. \%, p-\right.$.)

Heterogeneidade entre os grupos: $p$ - 0,000

Geral (|^2 - 98,42\%, p - 0,00);

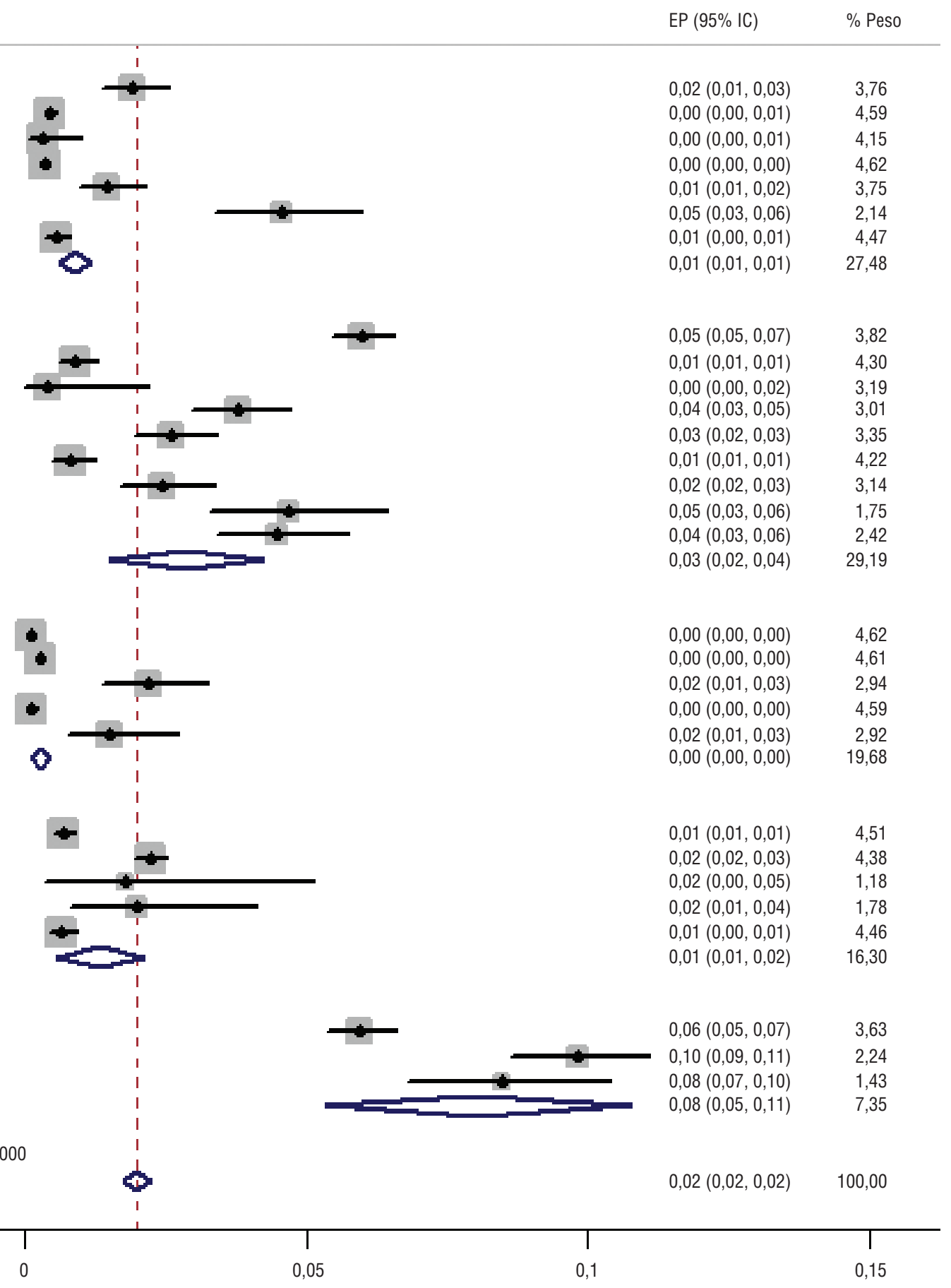

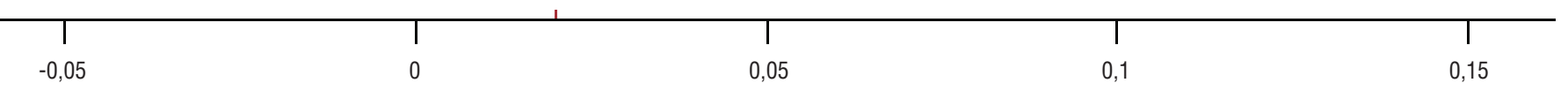

a EP: estimativa de prevalência; 95\%IC: intervalo de confiança de 95\%.

da comunidade, quando necessário (4) representam medidas potencialmente efetivas. Sua implementação é, no entanto, muitas vezes dificultada por restrições de recursos específicas para a configuração da prisão. No entanto, a ênfase deve sempre ser colocada no controle da transmissão da tuberculose, especialmente em períodos de crescimento das populações carcerárias e também da multirresistência a medicamentos (2), um vez que a transmissão da tuberculose é predominantemente intrainstitucional (26).
Melhorar o controle da tuberculose nas prisões pode beneficiar a comunidade em geral. As políticas públicas e os esforços comunitários de controle da doença não podem ignorar a tuberculose nas prisões (18). Os principais obstáculos para enfrentar essa situação são, de fato, 
políticos e sociais. A inércia do poder estatal é retroalimentada por um contexto social e cultural marcado por forte estigma, discriminação e preconceito em relação à população carcerária (43).

$\mathrm{O}$ presente trabalho tem limitações. A maioria dos estudos incluídos foi do tipo transversal, fornecendo apenas a prevalência pontual da situação em um determinado momento no tempo, sem capturar a natureza dinâmica da população prisional. Os métodos de rastreio e diagnóstico de tuberculose também foram variados entre estudos, o que pode ter afetado os resultados agrupados. Além disso, este trabalho baseou-se apenas em estudos publicados com revisão por pares nas bibliotecas MEDLINE, LILACS e SciELO. Portanto, dados importantes podem ter sido perdidos, de estudos não publicados e publicações cinzentas. A presença desse viés de publicação foi identificada por meio de gráfico de funil e do teste de Egger. Estudos com menor prevalência de tuberculose na população privada de liberdade poderiam ter sido encontrados se outras bibliotecas eletrônicas como a EMBASE, que enfoca a literatura europeia, tivessem sido incluídas na análise, o que consequentemente diminuiria o viés e a alta heterogeneidade encontrada no presente estudo.

Ademais, as variáveis incluídas na metarregressão não foram suficientes para explicar a heterogeneidade entre os estudos. Outras variáveis importantes, como a proporção de indivíduos do sexo feminino na amostra, coinfecção de M, Fedeli U, Scano F. Tuberculosis incidence in prisons: a systematic review. PLoS Med. 2010;7(12):e1000381.

2. Dara M, Acosta CD, Melchers NV, AlDarraji HA, Chorgoliani D, Reyes H, et al. Tuberculosis control in prisons: current situation and research gaps. Int J Infect Dis. 2015;32:111-7.

3. Droznin M, Johnson A, Johnson AM. Multidrug resistant tuberculosis in prisons located in former Soviet countries: A systematic review. PLoS One. 2017;12(3): e0174373.

4. Niveau G. Prevention of infectious disease transmission in correctional settings: a review. Public Health. 2006;120:33-41.

5. Abebe DS, Bjune G, Ameni G, Biffa D, Abebe F. Prevalence of pulmonary tuberculosis and associated risk factors in Eastern Ethiopian prisons. Int J Tuberc Lung Dis. 2011;15(5):668-73. tuberculose e HIV, presença de equipes de saúde no presídio, existência de triagem de tuberculose na entrada do sistema penitenciário e superlotação não puderam ser incluídas na análise pela escassez de dados nos estudos.

Outra limitação do presente estudo diz respeito à utilização da escala de Loney et al. (20) para avaliar a qualidade dos estudos incluídos na metanálise. Essa escala pode ter limitações uma vez que atribui pesos iguais a cada item da escala. O emprego de escalas para avaliação quantitativa permite que as pontuações sejam usadas como fator de ponderação na análise estatística dos resultados de uma metanálise, atribuindo-se maior peso aos trabalhos de melhor qualidade. No entanto, é discutível se essa pontuação é realmente representativa, permitindo comparar a qualidade metodológica dos artigos científicos. A quantificação implica em estabelecer um peso para cada item da escala, o qual é incerto, por não ser possível a comparação com um padrão-ouro para aferir sua validade. As ponderações usadas nas escalas se mostraram inconsistentes em revisões sistemáticas (47).

\section{CONCLUSÃO}

O presente estudo aponta e reafirma a magnitude da tuberculose entre a população privada de liberdade no contexto mundial. Também foi possível observar que a prevalência de tuberculose na população geral influencia a prevalência da

\section{REFERÊNCIAS}

6. Öngen G, Börekçi S, İçmeli OS, Birgen N, Karagül G, Akgün S, et al. Pulmonary tuberculosis incidence in Turkish prisons: importance of screening and case finding strategies. Tuberk Toraks 2013;61(1):21-7.

7. Aguilera XP, González C, Nájera-De Ferrari M, Hirmas M, Delgado I, Olea A. Tuberculosis in prisoners and their contacts in Chile: estimating incidence and latent infection. Int J Tuberc Lung Dis. 2016;20(1):63-70.

8. Habeenzu C, Mitarai S, Lubasi D, Mudenda V, Kantenga T, Mwansa T, et al. Tuberculosis and multidrug resistance in Zambian prisons, 2000-2001. Int J Tuberc. Lung Dis. 2007;11(11):1216-20.

9. Sanchez A, Gerhardt G, Natal S, Capone D, Espinola A, Costa W, et al. Prevalence of pulmonary tuberculosis and comparative evaluation of screening strategies in a Brazilian prison. Int J Tuberc Lung Dis. 2005;9(6):633-9. doença dentro dos presídios. Torna-se necessário elaborar e implementar políticas públicas para controle das altas taxas de tuberculose nesse grupo.

O controle da tuberculose na população privada de liberdade se estende além das paredes da prisão; afeta a população civil, já que familiares, funcionários da prisão e membros do sistema judiciário podem ser portas potenciais de saída para a transmissão da tuberculose. Medidas como o fortalecimento dos centros de saúde das prisões, com mão-de-obra e infraestrutura, triagem periódica e no momento do ingresso no presídio para a detecção de casos ativos e infecção latente, treinamento para prisioneiros e funcionários das prisões sobre prevenção de infecção por tuberculose, suporte ao diagnóstico e tratamentos imediatos são indicados para diminuir a carga da tuberculose e impedir que as prisões se mantenham como reservatório dessa doença. Atrair atenção e recursos para o problema da tuberculose nas prisões pode levar a uma melhora geral nas condições das prisões, na saúde dos reclusos e nos direitos humanos.

Conflitos de interesse. Nada declarado pelos autores.

Declaração. As opiniões expressas no manuscrito são de responsabilidade exclusiva dos autores e não refletem necessariamente a opinião ou política da RPSP/PAJPH ou da Organização PanAmericana da Saúde (OPAS).
10. Kuhleis D, Ribeiro AW, Costa ERD, Cafrune PI, Schmid KB, Costa LL, et al. Tuberculosis in a southern Brazilian prison. Mem Inst Oswaldo Cruz. 2012;107(7):909-15.

11. Telisinghe L, Fielding KL, Malden JL, Hanifa Y, Churchyard GJ, Grant AD, et al. High tuberculosis prevalence in a South African prison: the need for routine tuberculosis screening. PLoS One. 2014;9(1): e87262.

12. Valença MS, Scaini JLR, Abileira FS, Gonçalves CV, von Groll A, Silva PEA. Prevalence of tuberculosis in prisons: risk factors and molecular epidemiology. Int J Tuberc Lung Dis. 2015;19(10):1182-7.

13. Aerts A, Habouzit M, Mschiladze L, Malakmadze N,Sadradze N, Menteshashvili $\mathrm{O}$, et al. Pulmonary tuberculosis in prisons of the ex-USSR state Georgia: results of a nation-wide prevalence survey among sentenced inmates. Int J Tuberc Lung Dis. 2000;4(12):1104-10. 
14. Pendzich J, Maksymowicz-Mazur W, Pawłowska J, Filipczyk L, Kulawik I, Zientek J, et al. Tuberculosis among the homeless and inmates kept in custody and in penitentiary institutions in the Silesia region. Pneumonol Alergol Pol. 2015;83(1): 23-9.

15. Paião DSG, Lemos EF, Carbone ASS, Sgarbi RVE, Laranjeira Junior A, da Silva FM, et al. Impact of mass-screening on tuberculosis incidence in a prospective cohort of Brazilian prisoners. BMC Infect Dis. 2016;16:533.

16. Ali S, Haileamlak A, Wieser A, Pritsch M, Heinrich N, Loscher T, et al. Prevalence of pulmonary tuberculosis among prison inmates in Ethiopia, a cross-sectional study. PLoS One. 2015;10(12):e0144040.

17. Zerdo Z, Medhin G, Worku A, Ameni G. Prevalence of pulmonary tuberculosis and associated risk factors in prisons of Gamo Goffa Zone, South Ethiopia: a cross-sectional study. Am J Health Res. 2014;2(5):291-7.

18. World Health Organization (WHO). The end TB strategy. Genebra: WHO; 2015. Disponível em: http://www.who.int/tb/ End_TB_brochure.pdf?ua=1 Acessado em outubro de 2018.

19. Loney PL, Chambers LW, Bennett KJ, Roberts JG, Stratford PW. Critical appraisal of the health research literature: prevalence or incidence of a health problem. Chronic Dis Can. 1998;19(4):170-6.

20. Rodrigues CL, Ziegelmann PK. Metanálise: um guia prático. Rev HCPA. 2010;30(4): 436-47.

21. Knapp G, Hartung J. Improved tests for a random effects meta-regression with a single covariate. Stat Med. 2003;22(17): 2693-710.

22. Sterne JA, Sutton AJ, Ioannidis JP, Terrin $\mathrm{N}$, Jones DR, Lau J. Recommendations for examining and interpreting funnel plot asymmetry in meta-analyses of randomised controlled trials. BMJ. 2011;343:d4002.

23. Galvão TF, Pansani TSA, Harrad D. Principais itens para relatar revisões sistemáticas $\mathrm{e}$ meta-análises: a recomendação PRISMA. Epidemiol Serv Saude. 2015;24(2):335-42.

24. Banda HT, Gausi F, Harries AD, Salaniponi FM. Prevalence of smear-positive pulmonary tuberculosis among prisoners in Malawi: a national survey. Int J Tuberc Lung Dis. 2009;13(12):1557-9.

25. Banu S, Hossain A, Uddin MK, Uddin MR, Ahmed T, Khatun R, et al. Pulmonary tuberculosis and drug resistance in Dhaka Central Jail, the largest prison in Bangladesh. PLoS ONE. 2010;5(5):e10759.
26. Carbone ASS, Paião DSG, Sgarbi RVE, Lemos EF, Cazanti RF, Ota MM, et al. Active and latent tuberculosis in Brazilian correctional facilities: a cross-sectional study. BMC Infect Dis. 2015;15:24.

27. Chigbu LN, Uroegbu CU. Incidence and spread of mycobacterium tuberculosisassociated infection among aba federal prison inmates in Nigeria. J Health Popul Nutr. 2010;28(4):327-32.

28. Estevan AO, Oliveira SMVL, Croda J. Active and latent tuberculosis in prisoners in the Central-West Region of Brazil. Rev Soc Bras Med Trop. 2013;46(4):515-8.

29. Fuge TF, Ayanto SY. Prevalence of smear positive pulmonary tuberculosis and associated risk factors among prisoners in Hadiya Zone prison, Southern Ethiopia. BMC Res Notes. 2016;9:201.

30. Hanau-Berçot B, Grémy I, Raskine L, Bizet J, Gutierrez MC, Boyer-Mariotte S, et al. A one-year prospective study (1994-1995) for a first evaluation of tuberculosis transmission in French prisons. Int J Tuberc Lung Dis. 2000;4(9):853-9.

31. Henostroza G, Topp SM, Hatwiinda S, Maggard KR, Phiri W, Harris JB. The high burden of tuberculosis (TB) and human immunodeficiency virus (HIV) in a large Zambian Prison: a public health alert. PLoS ONE. 2013;8(8):e67338.

32. Jittimanee $S X$, Ngamtrairai $N$, White $M C$, Jittimanee S. A prevalence survey for smear-positive tuberculosis in Thai prisons. Int J Tuberc Lung Dis. 2007;11(5): 556-61.

33. Kazi AM, Shah AS, Jenkins CA, Shepherd $\mathrm{BE}$, Vermund $\mathrm{SH}$. Risk Factors and prevalence of tuberculosis human immunodeficiency virus, syphilis, hepatitis B virus, among prisoners in Pakistan. Int J Infect Dis. 2010;14 Suppl 3:e60-6.

34. Leung CC, Chan CK, Tam CM, Yew WW, $\mathrm{Kam} \mathrm{KM}, \mathrm{Au} \mathrm{KF}$, et al. Chest radiograph screening for tuberculosis in a Hong Kong prison. Int J Tuberc Lung Dis. 2005;9(6): 627-32.

35. Martin V, Guerra JM, Cayla JA, Rodriguez JC, Blanco MD, Alcoba M. Incidence of tuberculosis and the importance of treatment of latent tuberculosis infection in a Spanish prison population. Int J Tuberc Lung Dis. 2005;5(10):926-32.

36. Moges B, Amare B, Asfaw F, Tesfaye W, Tiruneh M, Belyhun Y, et al. Prevalence of smear positive pulmonary tuberculosis among prisoners in North Gondar Zone Prison, northwest Ethiopia. BMC Infect Dis. 2012;12:352.
37. Nogueira PA, Abrahão RMCM, Galesi VMN. Tuberculosis and latent tuberculosis in prison inmates. Rev Saude Publica. 2012;46(1):119-27.

38. Rao NA. Prevalence of pulmonary tuberculosis in Karachi central prison. J Pak Med Assoc. 2004;54(8):413-5.

39. Sretrirutchai S, Silapapojakul K, Palittapongarnpim P, Phongdara A, Vuddhakul V. Tuberculosis in Thai prisons: magnitude, transmission and drug susceptibility. Int J Tuberc Lung Dis. 2002;6(3): 208-14.

40. Winetsky DE, Almukhamedov O, Pulatov D, Vezhnina N, Dooronbekova A, Zhussupov B. Prevalence, risk factors and social context of active pulmonary tuberculosis among prison inmates in Tajikistan. PLoS One. 2014;9(1):e86046.

41. Melese A, Demelash H. The prevalence of tuberculosis among prisoners in Ethiopia: a systematic review and meta-analysis of published studies. Arch Public Health 2017;75:37.

42. Biadglegne F, Rodloff AC, Sack U. Review of the prevalence and drug resistance of tuberculosis in prisons: a hidden epidemic. Epidemiol Infect. 2015;143(5):887-900.

43. Larouzé B, Ventura M, Sánchez AR, Diuana V. Tuberculose nos presídios brasileiros: entre a responsabilização estatal e a dupla penalização dos detentos. Cad Saude Publica. 2015;31(6): 1127-30.

44. Barreto ML. Desigualdades em saúde: uma perspectiva global. Cienc Saude Coletiva. 2017;22(7):2097-108.

45. Raviglione M, Marais B, Floyd K, Lönnroth K, Getahun H, Migliori GB, et al. Scaling up interventions to achieve global tuberculosis control: progress and new developments. 2012;379(9829):1902-13.

46. Bertolozzi MR, Takahashi RF, Hino $\mathrm{P}$, Litvoc M, França FOS. O controle da tuberculose: um desafio para a saúde pública. Rev Med (São Paulo). 2014;93(2):83-9.

47. Sanderson S, Tatt ID, Higgins JP. Tools for assessing quality and susceptibility to bias in observational studies in epidemiology: a systematic review and annotated bibliography. Int $\mathrm{J}$ Epidemiol. 2007;36(3):666-76.

Manuscrito recebido em 2 de março de 2018. Aceito em versão revisada em 28 de julho de 2018. 
ABSTRACT

Prevalence of tuberculosis in incarcerated populations: systematic review and meta-analysis

Keywords Tuberculosis; prisons; prevalence; meta-analysis.
Objective. To estimate the prevalence of tuberculosis among incarcerated populations. Method. A systematic review with meta-analysis was performed. The MEDLINE/ PubMed, SciELO, and LILACS databases were searched for articles published from January 1997 to December 2016. The following search terms were used: ("tuberculosis" $[\mathrm{MeSH}] \mathrm{OR}$ "tuberculosis"[TIAB]) AND ("prisons"[MeSH] OR "prisons"[TIAB]); or ("tuberculose" [DeCS] OU "tuberculose" [palavras] E "prisões" [DeCS] OU "prisões" [palavras]). The primary outcome was the prevalence of tuberculosis with $95 \%$ confidence interval $(95 \% \mathrm{CI})$. In the metanalysis, variables associated with tuberculosis prevalence in incarcerated populations in the univariate analysis $(P \leq 0,20)$ were included in the final multivariate model.

Results. Based on the 29 studies included in the metanalysis, 2,163 prisoners with tuberculosis were identified. The combined prevalence of tuberculosis among prisoners was $2 \%$ (95\%CI: 0.02-0.02). The prevalence among prisoners from countries with tuberculosis prevalence of $0-24$ per 100,000 general population was below $1 \%$ $(95 \% \mathrm{CI}=0.00-0.00)$. In countries with overall tuberculosis prevalence of 25-99/100,000, the estimate among prisoners was 3\% $(95 \% \mathrm{CI}=0.02-0.04)$; and in countries with overall prevalence $\geq 300 / 100$ thousand, the estimated prevalence among prisoners was $8 \%$ $(95 \% \mathrm{CI}=0.05-0.11)$.

Conclusion. The present results support the notion of a high prevalence of tuberculosis among incarcerated populations worldwide. The results also show a link between the prevalence of tuberculosis in the general and the prevalence of tuberculosis in prisons.
RESUMEN

\section{Prevalencia de tuberculosis en la población privada de libertad: revisión sistemática y metanálisis}

Objetivo. Estimar la prevalencia de tuberculosis en la población privada de libertad. Métodos. Se realizó una revisión sistemática con metanálisis. Se seleccionaron estudios publicados desde enero de 1997 hasta diciembre del 2016 en las bases de datos MEDLINE/PubMed, SciELO y LILACS. Los términos de búsqueda fueron ("tuberculosis"[MeSH] OR "tuberculosis"[TIAB]) AND ("prisons"[MeSH] OR "prisons"[TIAB]); o ("tuberculose" [DeCS] OU "tuberculose" [palavras] E "prisões" [DeCS] OU "prisões" [palavras]). El resultado principal fue la prevalencia de tuberculosis con un intervalo de confianza de 95\% (IC95\%). En el metanálisis, las variables asociadas con la prevalencia de la tuberculosis en la población privada de libertad en el análisis univariado $(P \leq 0,20)$ se incluyeron en el modelo final multivariado.

Resultados. Con base en los 29 estudios incluidos en el metanálisis, se detectaron 2163 presos con tuberculosis. La prevalencia combinada de tuberculosis en los presos fue de $2 \%$ (IC95\%: 0,02-0,02). La prevalencia de tuberculosis en presos de los países con prevalencia de 0 a 24 por 100.000 habitantes en la población general fue inferior a $1 \%(\mathrm{IC} 95 \%=0,00-0,00)$. En los países con una prevalencia de tuberculosis de 25 a 99 por 100.000, la estimación fue de 3\% (IC95\% = 0,02-0,04); y en los países con prevalencia $\geq 300$ por 100.000 , de $8 \%$ (IC95\% = 0,05-0,11).

Conclusión. El presente estudio reafirma la alta prevalencia de la tuberculosis en la población privada de libertad en el contexto mundial. Los resultados muestran además una conexión entre la prevalencia de la tuberculosis en la población general y la observada dentro de los presidios.

Palabras-clave Tuberculosis; cárceles; prevalencia; meta-análisis. 